\title{
Mutation Profiling in Mitochondrial D-Loop Associated with Stomach Cancer and Tobacco Consumers
}

Rebecca Lalmuanpuii', Souvik Ghatak ${ }^{2}$, Jeremy L Pautu ${ }^{3}$, Doris Lallawmzuali ${ }^{3}$, Rajendra Bose Muthukumaran ${ }^{1}$ and Nachimuthu Senthil Kumar $^{2 *}$

${ }^{1}$ Department of Chemistry, Mizoram University, Aizawl, Mizoram, India

${ }^{2}$ Department of Biotechnology, Mizoram University, Aizawl, Mizoram, India

${ }^{3}$ Mizoram State Cancer Institute, Zemabawk, Aizawl, Mizoram, India

\begin{abstract}
Within North East India, the people of Mizoram are mongoloid in origin and Cancer is a major disease condition among this tribal populace. A peculiar habit of consuming "tuibur" (tobacco smoke-infused aqueous solution) has been practiced in Mizoram. Blood and oral swab samples were collected from stomach cancer patients, tuibur consumers and healthy people. DNA was extracted followed by PCR amplification of the D-loop region of mtDNA. Restriction enzyme digestion of $1050 \mathrm{bp}$ of the hyper variable control region of mtDNA was performed in order to gain an insight into the phylogenetic relationship of populace of Mizoram besides the genetic variations among tuibur consumers. The phylogram based on restriction enzyme analysis (Alul, Haell, $\mathrm{Mspl}$ and $\mathrm{Kpnl}$ ) of the D-loop region subsumed within same mtDNA haplogroups and the markers resulted in a similar clustering of the population. The polymorphic samples were sequenced, analyzed and compared with the MITOMAP database. In the hypervariable control region, 292A $>A A$ and $316 C>C C$ are novel microsatellites instability as they have been reported for the first time as it has not been found in the mitochondrial database. 292A>AA position of MT-HV II region is a locus for the mtTF1 binding site $Y$. Due to the microsatellite instability of this position, the binding of mtTF1 may be altered. $316 \mathrm{C}>\mathrm{CC}$ is present at the conserved sequence block II in MT-HV II region of human mitochondrial control region. The present study revealed a variety of mtDNA D-loop region mutations and polymorphisms among tuibur consumer besides stomach cancer patients of Mizoram, some of which might be involved in the development of carcinogenesis in stomach for the tuibur consumer.
\end{abstract}

Keywords: Mitochondrial DNA; Tuibur; Mizo- mongoloid; Polymorphisms; Phylogram

\section{Introduction}

The epidemiologic investigations have implicated various putative risk factors to the incidence of stomach cancer, albeit the etiology still remains unclear. However, it has been possible to identify certain risk factors that may contribute significantly to the cause of cancer leading to death. Most important among them are lifestyle habits, viz, tobacco consumption (in diverse forms) and diet. These two factors are especially important in the context of prevalence of various kinds of cancer [1]. While it is clear that diverse genetic pathways exist for the development and progression of various cancers, the emerging evidence strongly suggests that the pathogenesis of cancer is complex and multifactorial, ranging from the environment and dietary habits to genetic and molecular alterations [2].

Mitochondrial DNA is relatively more vulnerable to oxidative damage and it also exhibits higher mutation rate than nuclear DNA (nDNA) as a manifestation of the absence of protective histone proteins, limited DNA repair mechanisms as well as a high rate of Reactive Oxygen Species (ROS) generation due to oxidative phosphorylation [3]. The displacement loop (D-loop) is the only non-coding region (nucleotide position (np) 16024-576=1124 bp) of mitochondrial genome and is known to accumulate mutations at higher frequency than other regions [4]. It is a hot spot for mtDNA alterations comprising of two hyper variable regions (HVR) which are HVR1: nucleotide position 1602416383 and HVR2: nucleotide position 57-333. In addition, D-loop contains crucial elements for replication and transcription of mtDNA [5]. Hence, sequence alterations in D-loop region may contribute to impaired replication and/or transcription of mitochondrial genes which may impede the overall mitochondrial function and exacerbates cellular ROS generation. Accumulation of D-loop alterations has been implicated in several complex human diseases [5-7]. D-loop region of the mtDNA is the most potent accumulation site for many of these mutations as numerous polymorphisms have also been reported in this region as it does not contain protective histones, exhibits high oxidative stress associated with deficient DNA repair mechanisms [8]. D-loop is the only non-coding mtDNA region which possesses crucial elements for replication and transcription. Thus, the sequence alterations of this region may contribute to altered replication or transcription properties $[9,10]$.

Stomach cancer is the second leading cause of cancer death worldwide. In India, incidence of stomach cancer is the highest in Aizawl district of Mizoram with an age-adjusted rate (AAR) of 55.4 (male) and 24.4 (female) per $10^{5}$ populations [11]. In fact, the incidence is comparable and in some cases higher than Japan, Korea, Chile and Costa Rica where these cancers were traditionally considered to be high [1]. The people of Mizoram are culturally and ethnically distinct from the other tribes and communities of India. A peculiar habit of consumption of "tuibur" (tobacco smoke-infused aqueous solution) has been observed in Mizoram. Therefore, correlation of consumption of tobacco with the incidence of stomach cancer in Mizoram cannot be

*Corresponding author: Nachimuthu Senthil Kumar, Department of Biotechnology, Mizoram University, Aizawl - 796 004, Mizoram, India, Tel: 09436352574; E-mail: nskmzu@gmail.com

Received June 29, 2015; Accepted August 26, 2015; Published September 04, 2015

Citation: Lalmuanpuii R, Ghatak S, Pautu JL, Lallawmzuali D, Muthukumaran RB, et al. (2015) Mutation Profiling in Mitochondrial D-Loop Associated with Stomach Cancer and Tobacco Consumers. J Clin Med Genom 3: 122. doi: 10.4172/2472128X.1000122

Copyright: ( 2015 Lalmuanpuii R, et al. This is an open-access article distributed under the terms of the Creative Commons Attribution License, which permits unrestricted use, distribution, and reproduction in any medium, provided the original author and source are credited. 
ruled out [12]. The present study was undertaken to elicit the D-Loop gene polymorphism in stomach cancer patients and correlating with tobacco consumption in the 'liquid form' as tuibur.

\section{Materials and Methods}

\section{Sampling}

Blood samples were collected from 40 stomach cancer patients from Mizoram State Cancer Institute (Mizoram, India). Buccal swab samples were collected from 40 tuibur consumers and 40 non-tuibur consumers (without record of any disease). All volunteers were fully informed about the study and participated with their full consent. Detailed information on demographic factors, dietary habits, previous disease history, physical activity, tobacco habit, alcohol consumption, weight, and family history were collected from the study subjects using a standardized structured questionnaire. The age distribution of the three groups (tuibur consumer, control, and cancer patients) is ranging from 19 to 95,19 to 74 , and 40 to 78 years, respectively. The undertaken protocol were reviewed and approved by the Institutional Review Board of all institutes involved in the study. All sample were immediately stored under appropriate condition until further use.

\section{DNA extraction and PCR amplification}

DNA was extracted from the samples by following the modified protocol of Ghatak et al. (2013) [13]. The extracted DNA was dissolved in 1XTE buffer ( $\mathrm{pH} 8.0$ ) and stored at $-20^{\circ} \mathrm{C}$ until used. DNA extracts was amplified for the mtDNA D-loop region by using primers HMt-F (5'CACCATTAGCACCCAAAGCT-3') and HMt-R (5'-CTGTTAAAAGTGCATACCGCCA-3') as described by Salas et al. (2001) [14] for the HVI region. PCR (vapo.protect; Eppendorf) was carried out in $25 \mu \mathrm{l}$ total reaction volumes (containing $100 \mathrm{ng}$ template DNA, $0.2 \mathrm{pM}$ of each primer, $1 \mathrm{X}$ PCR buffer, $1.5 \mathrm{mM} \mathrm{MgCl}, 200$ $\mathrm{mM}$ dNTPs, 1 unit Taq DNA polymerase). The reaction mixture was heated to $94^{\circ} \mathrm{C}$ for $5 \mathrm{~min}$, followed by 40 cycles, each consisting of 1 min denaturation at $94^{\circ} \mathrm{C}, 1 \mathrm{~min}$ annealing at $63^{\circ} \mathrm{C}, 1.5 \mathrm{~min}$ extension at $72^{\circ} \mathrm{C}$, and a final 10 -min extension at $72^{\circ} \mathrm{C}$. The PCR amplification products $(10 \mu \mathrm{l})$ were subjected to electrophoresis (Bio-Rad) on $1.2 \%$ agarose gel in 1X Tris-acetate-EDTA buffer at $80 \mathrm{~V}$ for $30 \mathrm{~min}$ and stained with ethidium bromide (Himedia) and images were obtained in gel documentation (G-Box; Syngene, UK) system.

\section{Restriction digestion of the PCR product}

Restriction fragment-length polymorphism (RFLP) of the D-loop region was performed to check the polymorphism pattern. PCR products were digested with AluI, HaeIII, MspI and KpnI (Fermentas, Thermo Scientific) in a total volume of $10 \mu \mathrm{l}(2 \mu \mathrm{l}$ PCR products, $1 \mu \mathrm{l}$ enzyme buffers, $1 \mathrm{U}$ enzymes) and placed in the incubator at $37^{\circ} \mathrm{C}$ for 4 $\mathrm{h}$. The restriction products were analyzed by electrophoresis (Bio-Rad) on a $10 \%$ polyacrylamide gel and the molecular weight of restricted fragments was analyzed by gel documentation system (G-Box; Syngene) after ethidium bromide (Himedia) staining [15].

\section{Sequence and statistical analysis}

PCR products (selected on the basis of polymorphism pattern) were sequenced from both the directions to ensure reading accuracy. The sequences are submitted in the EBI repository database (EBI Accession No. LN558427 - LN558437). Sequences and chromatograms obtained were examined using chromas software version 2.13 and aligned by BLAST (http:// www.ncbi.nlm.nih.gov/blast). Sequences were aligned using CLUSTAL W and mutations were noted by using MEGA software ver 5.0. All sequences were compared with the latest version of Revised Cambridge Reference Sequence (rCRS) of the human mitochondrial DNA (NC_012920) and subsequently analyzed for the variation in sequences using Mito Tool Programming. The results of DNA sequence analysis were compared with the published Cambridge Sequence using Mutation Surveyor version 1.4 DNA mutation analysis software (Softgenetics, State College, PA). Sequence differences between tuibur consumer, stomach cancer and healthy blood samples were recorded as mtDNA polymorphisms. Each polymorphism was then verified against the Mitomap database (http://www.mitomap.org/) and further classified as novel or reported, depending on whether or not it is recorded in the database. Genetic relationship analyses, based on the RFLP pattern, of stomach cancer, control and tuibur consumers were performed using NETWORK 4.6.1.2. All mtDNA sequences were aligned by Clustal $\mathrm{W}$ program according to the rCRS. MEGA 5.05 program was used to calculate the distance matrix [16]. Frequencies of D-loop polymorphism among the various groups were tested for Hardy-Weinberg equilibrium by a chi-square $\left(\chi^{2}\right)$ test with one degree of freedom (df). The polymorphisms within each group were estimated using odds ratios (ORs) and 95\% confidence intervals (CIs). Logistic regression analyses were performed to compute the influence of tuibur consumption on stomach cancer risk. For all tests, a two-sided $\mathrm{P}$-value $<0.05$ was considered statistically significant.

\section{D-Loop secondary structure determination}

Secondary structure of the mitochondrial control region was inferred from the D-loop sequences [17]. The application predicts the structure based on the free energy parameters and the enthalpies were measured at $37^{\circ} \mathrm{C}$. However, they are assumed to be constant within the range of temperatures that might occur in vivo or in the laboratory. This enables the server to extrapolate free energies to other temperatures and to fold at these temperatures.

\section{Results}

PCR products for mtDNA D-loop region on agarose gel showed a fragment of about $1050 \mathrm{bp}$. Digestion of amplified fragments of mtDNA D-loop region by HaeIII, AluI, MspI and KpnI restriction endonucleases along with their rate of frequency are listed in Figure 1. Eight restriction patterns for AluI enzyme (with 5 heteroplasmy from tuibur and 2 heteroplasmy from cancer) and seven restriction patterns for HaeIII enzyme (with 3 heteroplasmy from tuibur consumer tuibur and 2 heteroplasmy from cancer) in Mizoram population were observed. The four chosen restriction enzymes exhibited 21 different polymorphic patterns. Moreover, MspI enzyme also showed two restriction patterns with 1 heteroplasmy variation in case of tuibur consumer, while four restriction patterns were revealed for KpnI enzyme. The network analysis showed frequency distribution of motifs in the population based on the PCR-RFLP product. Figure 1 also depicts that $37.5 \%$ of stomach cancer patient and $62.5 \%$ of tuibur consumers are clustering together.

A total of $27 \mathrm{mtDNA}$ D-loop sequence variations were observed in $25 \%$ of samples. In Table 1 shows the sequence variation of the mtDNA D-loop region of the two different groups (tuibur consumer and stomach cancer patient samples), sample frequency, nucleotide position, mutant type and the name of mutation in comparison with the healthy (control) group. Among the stomach cancer and tuibur consumer samples, it has been found that the type of mutations are mostly transition base substitution with $55.55 \% \mathrm{~T}>\mathrm{C}, \mathrm{C}>\mathrm{T}, \mathrm{A}>\mathrm{G}$ and $\mathrm{G}>\mathrm{A}$ base substitution in stomach cancer and $92.86 \% \mathrm{~T}>\mathrm{C}, \mathrm{C}>\mathrm{T}, \mathrm{A}>\mathrm{G}$ and $\mathrm{G}>\mathrm{A}$ base substitution in tuibur consumer. 


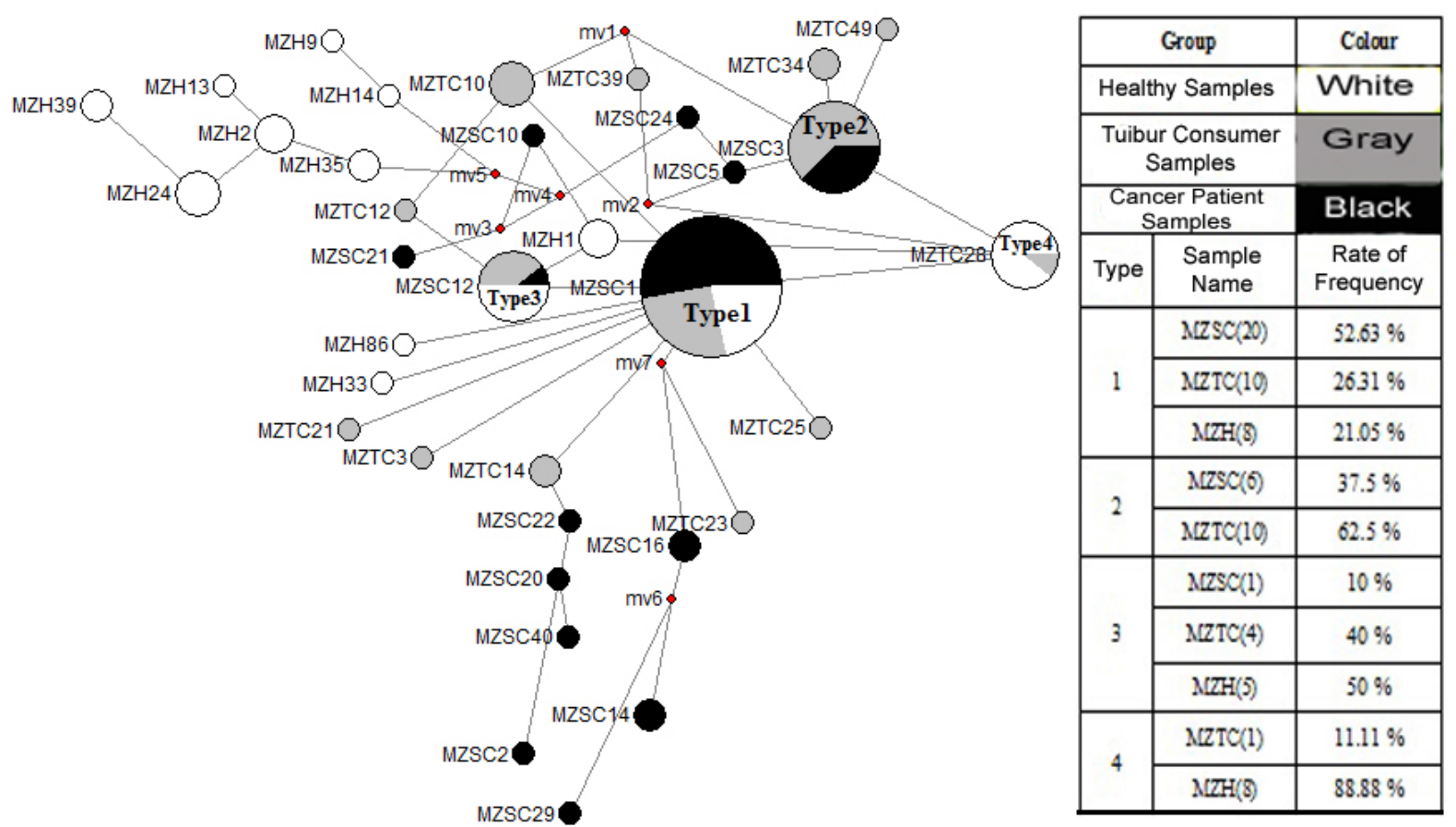

Figure 1: Phylogenetic network illustrating the genetic affinities among three different sample groups.

\begin{tabular}{|c|c|c|}
\hline Sample Name & Sample Frequency (\%) & Mutation position and type \\
\hline Sc 10,24 & 18.18 & $16051 A>G$ \\
\hline \multirow{3}{*}{ T 40} & \multirow{3}{*}{9.10} & $16092 T>C$ \\
\hline & & $94 \mathrm{G}>\mathrm{A}$ \\
\hline & & $214 A>G$ \\
\hline \multirow{6}{*}{ Sc 1} & \multirow{6}{*}{9.10} & $16093 \mathrm{~T}>\mathrm{C}$ \\
\hline & & $16266 \mathrm{C}>\mathrm{T}$ \\
\hline & & $16324 \mathrm{~T}>\mathrm{C}$ \\
\hline & & $16355 \mathrm{C}>\mathrm{T}$ \\
\hline & & $16391 \mathrm{G}>\mathrm{A}$ \\
\hline & & $* 292 A>A A$ \\
\hline \multirow{7}{*}{ T 1} & \multirow{7}{*}{9.10} & $16218 \mathrm{C}>\mathrm{T}$ \\
\hline & & $16289 A>G$ \\
\hline & & $16293 A>G$ \\
\hline & & $16526 \mathrm{G}>\mathrm{A}$ \\
\hline & & $183 A>G$ \\
\hline & & $184 G>A$ \\
\hline & & $185 \mathrm{G}>\mathrm{A}$ \\
\hline \multirow{2}{*}{ Sc 11} & \multirow{2}{*}{9.10} & $16234 \mathrm{C}>\mathrm{T}$ \\
\hline & & $153 A>G$ \\
\hline \multirow{3}{*}{$\begin{array}{c}\text { Sc } 3,31,33 \\
\text { T } 24\end{array}$} & \multirow{3}{*}{36.36} & $16290 \mathrm{C}>\mathrm{T}$ \\
\hline & & $16319 G>A$ \\
\hline & & $235 A>G$ \\
\hline \multirow{2}{*}{ Sc 10} & \multirow{2}{*}{9.10} & $16298 \mathrm{~T}>\mathrm{C}$ \\
\hline & & $16327 \mathrm{C}>\mathrm{T}$ \\
\hline Sc 11,24 & 18.20 & $150 C>T$ \\
\hline Sc $3,31,33$ & 27.30 & $151 C>T$ \\
\hline $\begin{array}{c}\text { Sc } 1 \\
\text { T1 }\end{array}$ & 18.20 & ${ }^{*} 316 \mathrm{C}>\mathrm{CC}$ \\
\hline
\end{tabular}

Sc- Stomach cancer, $T$ - Tuibur

*292A>AA and *316C>CC are novel mutations

Table 1: Variations in D-loop region of stomach cancer and tuibur consumers.
The DNA sequence analysis elicited a correlation between tuibur consumer and stomach cancer patient mtDNA control region (HVR1 and HVR2) genomics. Three mutations, viz., 16290C>T, 16319G $>$ A and $235 \mathrm{~A}>\mathrm{G}$ were observed in mostly tuibur consumers along with stomach cancer samples and hence we can conclude that these three potent mutations might have occurred in tuibur consumer samples as the manifestations of mutagenic effects of tuibur. 292A>AA mutation is a potential pathogenic mutation for stomach cancer patient due to the alteration of TF1 binding site $\mathrm{Y}$, while $316 \mathrm{C}>\mathrm{CC}$ mutation is also exhibited by both tuibur consumers and stomach cancer patient samples, indicative of the potent pathogenic mutation among both groups.

Unique properties of mtDNA D-loop that makes it a very valuable tool for both evolutionary and human identification studies include the high copy number, cytoplasmic inheritance, and rapid rate of evolution. The tuibur consumers and stomach cancer samples were found to cluster together showing close similarity (Figure 1). Bivariate analysis of the three groups (stomach cancer, tuibur consumer and healthy control) revealed that the mutation frequency due to consumption of tuibur (OR: 2.33 ; 95\% CI: $0.717-7.58$; $\mathrm{P}<0.0003$ ) was found to be a major risk factor for the development of gastric cancer.

The observed transition base substitutions were $16290 \mathrm{C}>\mathrm{T}$, $16319 \mathrm{G}>\mathrm{A}$ and $235 \mathrm{~A}>\mathrm{G}$ with maximum frequency of $36.37 \%$, besides a novel transition base insertion $(316 \mathrm{C}>\mathrm{CC})$ was also found. For tuibur consumers and stomach cancer patients, the D-Loop secondary structures were similar and relatively unrelated from the healthy control specimen based on the major loop (Figure 2). These repeats are mainly grouped in the Control Region (CR) extremities, following the mini-satellite and near the L strand $3^{\prime}$ region. Inverted repeats may be tandemly or closely arranged forming hairpin structures by use of complementarity the parts, such as 5'ATGTACGGTAAATGGCTTT3; 5'CCAAAAGATAAAATTTGAAA3', 5‘GGGGTGGCTT TGGAGTT3; 


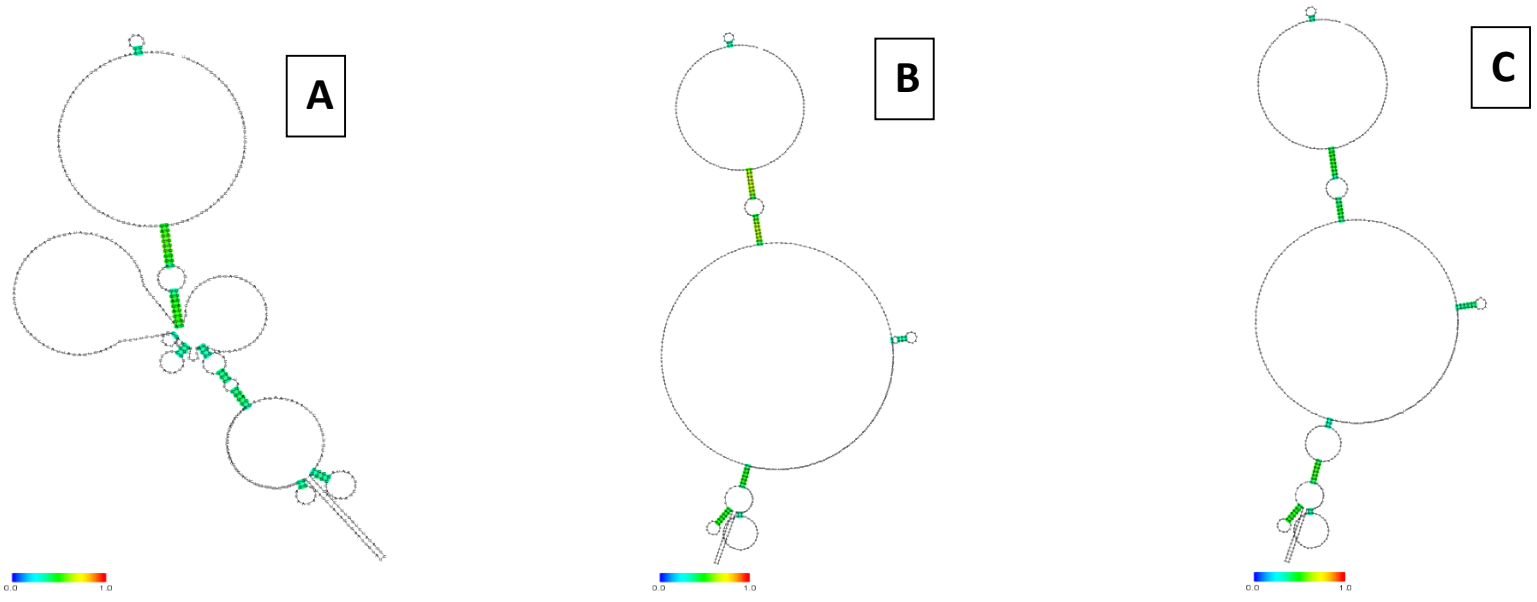

Figure 2: Secondary structures of Mitochondrial control region. (A) Healthy control, (B) Tuibur Consumer, (C) Stomach Cancer.

or separated by up to one hundred bases like 5'GGGGTGACTGTTAAAA GTG3'... 5'TTTTATGTACTACAGGT3؛ Other repeats, even imperfect ones, systematically form stem-loop structures using MFOLD software.

\section{Discussion}

Tuibur consumers and stomach cancer patient sequences have shown close relationship and hence clustered together. Buccal swab samples of healthy control and tuibur consumers along with blood samples of stomach cancer patient samples were also analyzed, in the present study, for the identification of sequence variation in the mtDNA D-loop region. Although, the investigation of all sequence data have exhibited characteristic variations, yet it provides the essential information for defining the macrohaplogroups such as D, A, M and R. Haplogroups-A, M were common in the population, in all type of samples (cancer, tuibur and normal) in this population. All the healthy samples could be placed in macrohaplogroups- $\mathrm{M}$ or $\mathrm{N}$ and in the case of stomach cancer and tuibur samples it was R, D and A, interestingly, the present data suggest novel haplogroups-A, D and R.

PCR-RFLP analysis is a simple and robust technique that can be used as a potential tool for early detection of biomarkers in the study of tobacco specific carcinogens induced stomach cancer [15]. The PCR-RFLP results have shown a significant level of heteroplasmy and polymorphic patterns at the D-loop region of mtDNA in Mizo population among the following groups: tuibur consumer, healthy (control) volunteer and stomach cancer patient. It is important to note that $292 \mathrm{~A}>\mathrm{AA}$ and $316 \mathrm{C}>\mathrm{CC}$ are novel base insertions (novel microsatellite instability) reported herein for the first time, as they have not been reported earlier in the mitochondrial database. 292A>AA position of MT-HV II region is a locus for the mtTF1 binding site $\mathrm{Y}$ and the microsatellite instability of this position is indicative of the impaired binding of mtTF1.316C >CC is present at the conserved sequence block II in MT-HV II region of human mitochondrial control region. Due to the presence of microsatellite instability at this position, the function of the mitochondrial control region may also be altered.

The mutation sites identified, in the present study, appeared to be predisposed towards mutation. The D-loop control region sites such as $309 \mathrm{C}>\mathrm{T}, 310 \mathrm{~T}>\mathrm{C}$ and $16223 \mathrm{C}>\mathrm{T}$ are observed in almost every stomach patient specimen analyzed as it demonstrates near confluence in this small cohort. The non-coding displacement (D)-loop, especially a mononucleotide repeat (poly-C) between 303 and 315 nucleotides
(D310), has been recently identified as a frequent hotspot for mutations in human neoplastic, including stomach cancer [18]. As such, this unique attribute may be considered as a potential biomarker for stomach cancer provided this behavior is consistent in transforming to blood. Functional studies are further needed to elucidate the biological significance of these mutant alleles in the tumorigenesis process of various tissues.

The search for the secondary structures also uncovered numerous stable inverted perfect or imperfect repeats that constantly turn into stable stem-loop structures when the DNA is stable and stranded for healthy control. It is worthwhile to note that all conserved segments depicted in fragments a-c form hairpin-like structures indicative of mirror like sequences for these conserved regions (e.g., inverted tandem repeats), but with the possible exception of healthy Control's CR, none of the CR (Stomach Cancer and Tuibur consumer) form stable secondary structures. Finally, two TCCC motifs exist in our sequence CR, but none of them are linked to the putative cloverleaf secondary structures. Taken together, confirmation rearrangements (CRs) of stomach cancer could not be divided into distinct conserved or variable domains, while tandem repeats of poly $\mathrm{C}$ stretch and conserved structural elements in the mid-region were observed. In the present study, we have observed that the $\mathrm{G}+\mathrm{C}$-rich region of stomach cancer and healthy (control) population besides a poly-C stretch is present which may be involved in transcriptional control or may be the site for initiation of replication that is consistent with the study reported by others [19]. For tuibur consumers and stomach cancer samples, the stem-loop secondary structures plus flanking sequences were completely identical and the stem was formed by a perfect match of 17 nucleotide pairs.

The state of Mizoram being one of the high risk/incidence regions of stomach cancer in India [20], few epidemiological studies have been carried out earlier to find out various potential risk factors for stomach cancer. It has been pointed out that the consumption of tuibur may be one of the important risk factors for the high prevalence of stomach cancer among the populace in Mizoram [12] which is consistent with the present study. Putative toxic effects of tuibur were also studied using modified version of Allium test [21-23]. Oxidative damage caused by smoking has also been shown to inhibit mitochondrial enzyme activity in platelets and cause mitochondrial dysfunction in alveolar macrophages [21]. In addition, an increase in mtDNA content and decline in mitochondrial function also occurs in response to DNA- 
Citation: Lalmuanpuii R, Ghatak S, Pautu JL, Lallawmzuali D, Muthukumaran RB, et al. (2015) Mutation Profiling in Mitochondrial D-Loop Associated with Stomach Cancer and Tobacco Consumers. J Clin Med Genom 3: 122. doi: 10.4172/2472-128X.1000122

damaging agents, including tobacco related product [22]. Moreover, a recent study showed that tuibur consumers have three times higher risk of stomach cancer than tuibur non-consumers [20,24]. Xenobiotic species such as 2-aminonaphthalene, 4 -aminobiphenyl, tobacco specific nitrosamines (TSNAs) which are thought to be responsible for many cancers [25], along with nicotine, nornicotine and myosmine might be also present in tuibur solution. As tuibur is not scientifically well characterized and only very limited health literature is available on the adverse effects of prolonged consumption of tuibur, the evaluation of potentially deleterious effects of tuibur solution on human health will be the focus of future research.

To our knowledge, the present work is an experimental study that has provided the types of mtDNA D-loop mutations in the development of stomach cancer with respect to the putative carcinogenetic effects of the tobacco smoke infused water (tuibur). The mtDNA analysis presented, in this study, has demonstrated that D-loop alterations may be helpful to identify individuals with tobacco consumption in general, and consumption of tuibur in particular, who are specifically at high risk for stomach cancer.

\section{Acknowledgements}

The authors thank the Department of Biotechnology (DBT), New Delhi, India for the infrastructural support in the form of Bioinformatics Infrastructure Facility and State Biotech Hub. We also thank all sample donors for their voluntary and invaluable support.

\section{Conflict of Interest}

The authors report no conflict of interests. The authors alone are responsible for the content and writing of the paper.

\section{References}

1. Mallath MK, Taylor DG, Badwe RA, Rath GK, Shanta V, et al. (2014) The growing burden of cancer in India: epidemiology and social context. Lancet Oncol 15: e205-212.

2. Tipirisetti NR, Govatati S, Pullari P, Malempati S, Thupurani MK, et al. (2014) Mitochondrial control region alterations and breast cancer risk: A study in South Indian population. PLoS One 9: e85363.

3. Croteau DL, Bohr VA (1997) Repair of oxidative damage to nuclear and mitochondrial DNA in mammalian cells. J Biol Chem 272: 25409-25412.

4. Michikawa Y, Mazzucchelli F, Bresolin N, Scarlato G, Attardi G (1999) Agingdependent large accumulation of point mutations in the human mtDNA control region for replication. Science 286: 774-779.

5. Clayton DA (2000) Transcription and replication of mitochondrial DNA. Hum Reprod 15 Suppl 2: 11-17.

6. Govatati S, Deenadayal M, Shivaji S, Bhanoori M (2013) Mitochondrial NADH: Ubiquinone oxidoreductase alterations are associated with endometriosis. Mitochondrion 13: 782-790.

7. Chen JB, Yang YH, Lee WC, Liou CW, Lin TK, et al. (2012) Sequence-based polymorphisms in the mitochondrial D-loop and potential SNP predictors for chronic dialysis. PLoS One 7: e41125.

8. Wang C, Zhang F, Fan H, Peng L, Zhang R, et al. (2011) Sequence polymorphisms of mitochondrial D-loop and hepatocellular carcinoma outcome. Biochem Biophys Res Commun 406: 493-496.

9. Penta JS, Johnson FM, Wachsman JT, Copeland WC (2001) Mitochondrial DNA in human malignancy. Mutat Res 488: 119-133.
10. Tan DJ, Bai RK, Wong LJ (2002) Comprehensive scanning of somatic mitochondrial DNA mutations in breast cancer. Cancer Res 62: 972-976.

11. National Cancer Registry Programme (NCRP) (2010) Three-year report of the population based cancer registries 2006-2008, (First report of 20 PBCRs in India), Bangalore. Indian Council Medical Research.

12. Phukan RK, Zomawia E, Narain K, Hazarika NC, Mahanta J (2005) Tobacco use and stomach cancer in Mizoram, India. Cancer Epidemiol Biomarkers Prev 14: $1892-1896$.

13. Ghatak S, Muthukumaran RB, Nachimuthu SK (2013) A simple method of genomic DNA extraction from human samples for PCR-RFLP analysis. J Biomol Tech 24: 224-231.

14. Salas A, Lareu MV, Carracedo A (2001) Heteroplasmy in mtDNA and the weight of evidence in forensic mtDNA analysis: a case report. Int J Legal Med 114 186-190.

15. Ghatak S, Lallawmzuali D, Mukherjee S, Mawia L, Pautu JL, et al. (2014) Polymorphism in mtDNA control region of Mizo-Mongloid Breast Cancer samples as revealed by PCR-RFLP analysis. Mitochondrial DNA.

16. Tamura K, Peterson D, Peterson N, Stecher G, Nei M, et al. (2011) MEGA5 Molecular evolutionary genetics analysis using maximum likelihood, evolutionary distance, and maximum parsimony methods. Mol Biol Evol 28:2731-2739

17. Zuker M (2003) Mfold web server for nucleic acid folding and hybridization prediction. Nucleic Acids Res 31: 3406-3415.

18. Xu C, Tran-Thanh D, Ma C, May K, Jung J, et al. (2012) Mitochondrial D310 mutations in the early development of breast cancer. Br J Cancer 106: 15061511.

19. Malakar M, Devi KR, Phukan RK, Kaur T, Deka M, et al. (2014) p53 codon 72 polymorphism interactions with dietary and tobacco related habits and risk of stomach cancer in Mizoram, India. Asian Pac J Cancer Prev 15: 717-723.

20. Phukan RK, Zomawia E, Hazarika NC, Narain K, Mahanta J, et al. (2004) High prevalence of stomach cancer among the people of Mizoram, India. Curr Sci 87: 285-286.

21. Smith PR, Cooper JM, Govan GG, Harding AE, Schapira AH (1993) Smoking and mitochondrial function: a model for environmental toxins. Q J Med 86: 657660

22. Lewis PD, Mehrotra D, Mahdi AA, Sarin R, Kowtal P, et al. (2002) Mitochondrial DNA mutations in the parotid gland of cigarette smokers and non-smokers. Mutat Res 18: 47-54.

23. Mahanta J, Chetia M, Hazarika NC, Narain K, Sharma SK (1998) Toxicity of tuibur, a unique form of tobacco smoke extract used in Mizoram, India. Curr Sci 75: $381-384$.

24. Malakar M, Devi KR, Phukan RK, Kaur T, Deka M, et al. (2012) Genetic polymorphism of glutathione S-transferases $\mathrm{M} 1$ and $\mathrm{T} 1$, tobacco habits and risk of stomach cancer in Mizoram, India. Asian Pac J Cancer Prev 13: 4725-4732.

25. Hecht SS (1999) Tobacco smoke carcinogens and lung cancer. J Natl Cancer Inst 91: 1194-1210. 